\section{Thrombolyse et infarctus cérébral aigu}

Halim Abboud, Pierre Amarenco
> Le devenir de l'infarctus cérébral s'est transformé avec l'arrivée de la thrombolyse, lorsque le patient peut être traité. Le rt-PA (recombinant tissue plasminogen activator), par son action thrombolytique, apparaît comme l'espoir de dissoudre des caillots et infléchir favorablement la destinée de l'ischémie cérébrale aiguë. Dans les indications et les contre-indications décrites dans l'étude NINDS (National institute of neurological disorders and stroke), et sous la responsabilité de neurologues entraînés, en raison des risques d'infarctus hémorragique associés, il est aujourd'hui recommandé de pratiquer la thrombolyse intraveineuse avec une dose de rt-PA de $0,9 \mathrm{mg} / \mathrm{kg}$ dans les trois premières heures. La thrombolyse par voie intra-artérielle, qui peut être pratiquée, sous certaines conditions, jusqu'à 6 heures après le début des symptômes, n'est praticable que dans les centres médicaux disposant d'un neuroradiologue interventionnel. Nous sommes au tout début du traitement de l'infarctus cérébral: la complexité et la diversité de ses mécanismes et de ses causes exigent l'intervention de neurologues entraînés, équipés d'IRM fonctionnant « 24 h sur 24 », de matériel d'examen par ultrasons et d'une table d'angiographie.

$<$

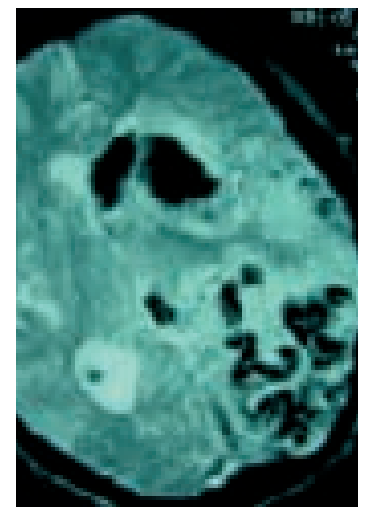

nase ou le rt-PA, par voie intraveineuse, contre placebo, ont été conduites. L'objectif de ces études était de mesurer l'efficacité de ces traitements sur l'amélioration du handicap fonctionnel. Les résultats de ces travaux ont permis à différentes sociétés savantes, dont la Société française neurovasculaire (SFNV), d'établir des recommandations quant à l'utilisation du traitement thrombolytique dans l'infarctus cérébral aigu [1].

\section{Thrombolyse intraveineuse par le rt-PA}

\section{Étude NINDS}

L'étude NINDS (National institute of neurological disorders and stroke) [2] s'est déroulée en deux parties. La première a évalué l'efficacité clinique du rt-PA $(0,9 \mathrm{mg} / \mathrm{kg})$ dans les 24 premières heures, mesurée par l'échelle de cotation de l'intensité du déficit neurologique des accidents vasculaires cérébraux (AVC) du National institute of health (NIH stroke scale, NIHSS). Près de la moitié (47\%) des patients dans le groupe rtPA présentèrent une amélioration rapide, contre $39 \%$ dans le groupe placebo. Le taux d'hémorragie cérébrale symptomatique fut de $6 \%$ dans le groupe traité (aucun dans le groupe placebo). La seconde partie de l'étude a évalué l'efficacité du rt-PA à trois mois sur la récupération neurologique (score NIHSS: 0 ou 1 ) et en termes de handicap fonctionnel (échelle modifiée de Rankin: 0 ou 1; score de Barthel: 95 ou 100). 
Le groupe traité par rt-PA avait un pourcentage de guérison significativement plus important que le groupe témoin. Le taux d'hémorragie cérébrale était nettement plus élevé dans le groupe rt-PA ( $7 \%$ contre $1 \%$ dans le groupe placebo). Toutefois, le taux de mortalité à trois mois fut inférieur (17\%) dans le groupe traité à celui du groupe témoin (21\%). Le gain thérapeutique était supérieur lorsque le traitement était administré avant 90 minutes [3].

En regard de ces résultats, une absence de handicap neurologique est attendu chez 160 patients pour 1000 patients traités avant la $3^{\mathrm{e}}$ heure. Les résultats de l'étude NINDS (1995) et l'approbation du rt-PA par la FDA (Food and drug administration) en juin 1996 ont permis la diffusion de la thrombolyse intraveineuse aux États-Unis.

\section{Autres essais}

L'European cooperative acute stroke study (ECASS I) [4] a concerné 620 patients présentant un infarctus cérébral datant de moins de 6 heures. Les critères d'évaluation primaires comportaient l'observation d'une différence de 15 points dans le score de Barthel et la baisse d'un niveau dans l'échelle modifiée de Rankin à 3 mois. Aucune différence n'était constatée sur les critères d'évaluation primaires. La mortalité à 30 jours était plus élevée dans le groupe traité (17,9\% contre $12,7 \%)$.

ECASS II [5] est un essai randomisé en double aveugle, contre placebo, du rt-PA moins de 6 heures après le début des symptômes. Les résultats n'ont pas montré de différence significative entre les deux groupes en ce qui concerne l'amélioration du handicap neurologique à 3 mois.

ATLANTIS (Alteplase thrombolysis for acute noninterventional therapy in ischemic stroke) [6] est un essai randomisé en double aveugle, contre placebo, du rt-PA à la dose de $0,9 \mathrm{mg} / \mathrm{kg}$ jusqu'à la $6^{\mathrm{e}}$ heure après le début des symptômes. L'étude a porté sur 613 patients et n'a pas démontré de bénéfice sur le handicap et la mortalité.

\section{Thrombolyse intraveineuse par la streptokinase}

Le Multicenter acute stroke trial-Italie (MAST-I) [7] est une étude multicentrique randomisée en double aveugle contre placebo, menée chez des patients souffrant d'un infarctus cérébral datant de moins de 6 heures. L'essai comportait 4 groupes de traitement: streptokinase 1,5 MU en perfusion intraveineuse sur 1 heure, aspirine $300 \mathrm{mg} / \mathrm{j}$ pendant 10 jours, aspirine + streptokinase, placebo. L'efficacité était évaluée par l'échelle de Rankin à 6 mois; 622 patients ont été inclus, avant l'interruption de l'essai en raison de l'augmentation de la mortalité. Au $10^{e}$ jour, il existait déjà une surmortalité dans le groupe des patients ayant reçu la streptokinase, surmortalité qui persistera au $6^{\mathrm{e}}$ mois et, plus encore, dans le groupe streptokinase + aspirine.
Le multicenter acute stroke trial-Europe (MAST- $\varepsilon$ ) [8] a inclus des patients souffrant d'un infarctus datant de moins de 6 heures. Les patients traités recevaient 1,5 MU de streptokinase en perfusion intraveineuse sur une heure. Cette étude fut stoppée par le comité de surveillance à partir des résultats observés chez les 270 premiers patients, en raison d'une surmortalité dans le groupe traité. La fréquence des hémorragies cérébrales symptomatiques était significativement plus élevée dans le groupe traité ( $18 \%$ contre $3 \%$ ).

L'Australian streptokinase trial (ASK) [9] a testé I'hypothèse selon laquelle la streptokinase administrée à la dose de 1,5 MU en perfusion par voie intraveineuse dans les 4 heures suivant un infarctus cérébral pouvait réduire la mortalité et la morbidité à 3 mois. Cette étude, qui a inclus 340 patients, a été interrompue en raison d'un surcroît d'effets indésirables et de surmortalité dans le groupe traité après la $3^{e}$ heure.

\section{En pratique : quel médicament utiliser, et à quelle dose?}

II n'existe pas d'étude permettant la comparaison directe entre le rt-PA et la streptokinase. Les essais cliniques européens et australien ayant pour but de tester la streptokinase ont été interrompus prématurément, après l'augmentation de la mortalité et des complications hémorragiques cérébrales chez les patients ayant reçu cet agent thrombolytique. II faut cependant souligner que, dans ces essais, un traitement antiagrégant et/ou anticoagulant était autorisé dans les 24 heures. 0 n ne peut donc savoir si l'absence d'efficacité du traitement avec un taux d'hémorragie intracrânienne augmenté est liée à la streptokinase ou à son association avec un autre traitement $[7,9]$. La méta-analyse des essais de streptokinase chez des patients traités dans les 3 heures montre d'ailleurs une efficacité comparable à celle du rt-PA même si, du fait du faible nombre de patient, les résultats n'étaient pas statistiquement significatifs. Il est cependant peu probable qu'un laboratoire se lance dans un essai avec la streptokinase dans les trois premières heures de l'ischémie cérébrale.

Quant aux résultats de l'étude NINDS, l'effet de la thrombolyse par le rt-PA n'est bénéfique que dans le sous-groupe où elle est pratiquée sans traitement associé dans les 24 premières heures.

Ainsi, il est aujourd'hui recommandé de pratiquer la thrombolyse intraveineuse avec une dose de rt-PA de 0,9 mg/kg dans les trois heures suivant le début des symptômes [1, 4, 10]. Dix pour cent de la dose totale sont injectés en 1 à 2 minutes, les $90 \%$ restants l'étant en 1 heure. Le profil du malade pouvant tirer bénéfice du traitement, sans risque de saignement excessif, n'est pas encore bien défini. Il est possible que des modifications des protocoles thérapeutiques concernant la dose ou le délai d'administration améliorent aussi la sécurité du traitement. 


\section{Peut-on établir des facteurs de risque d'hémorragie intracrânienne?}

L’hémorragie intracérébrale (HIC) est une complication potentiellement mortelle de la thrombolyse intraveineuse (Figure 1). Les facteurs de risque d'infarctus hémorragique ou d'hématome cérébral post-thrombolyse ne sont pas encore connus dans leur ensemble.

Dans la méta-analyse de J.M. Wardlow et al. [11], l'incidence d'une complication hémorragique symptomatique intracrânienne dans le groupe des patients thrombolysés était de $9,6 \%$ contre $2,6 \%$ dans le groupe des patients témoins. Ainsi, on peut conclure à une augmentation de $7 \%$ d'hémorragie intracrânienne symptomatique dans le groupe des patients thrombolysés. Dans le groupe de patients traités avant la $3^{\mathrm{e}}$ heure inclus dans les essais du rt-PA, une méta-analyse limitée à ces essais montre une augmentation du risque d'hémorragie intracrânienne dans le groupe des patients traités, mais moins important que dans le groupe des patients traités après 3 heures. Ce risque augmente avec la sévérité du déficit neurologique initial et avec l'importance des signes précoces d'ischémie observés au scanner cérébral. De même, l'âge, l'hypertension artérielle, l'hyperglycémie et l'occlusion embolique du segment $\mathrm{Ml}$ de l'artère cérébrale moyenne sont associés à un plus grand risque d'hémorragie intracrânienne après traitement avec le rt-PA $[10,12,13]$.

Des études centrées sur l'application des nouvelles séquences d'IRM au stade aigu de l'infarctus cérébral (coefficient apparent de diffusion $[C A D]$, perfusion) chez l'homme et dans le modèle animal suggèrent que l'IRM diffusion-perfusion pourrait aider à prévoir le risque d’hémorragie intracérébrale après thrombolyse [14]. Les modèles expérimentaux ont confirmé que les tissus ischémiques possédant les valeurs de CAD les plus basses sont associés aux signes histopathologiques ischémiques les plus graves $[15,16]$ et à une altération de la barrière hématoméningée $[17,18]$, et sont donc à un plus haut risque de saignement. Une corrélation entre hypoperfusion sévère initiale et transformation hémorragique a été mise en évidence à l'aide de la tomographie d'émission monophotonique dans la thrombolyse intra-artérielle [19, 20].

\section{Échecs de la thrombolyse}

Les causes d'échec sont multiples. En premier lieu, la localisation de l'occlusion semble être importante. Les occlusions de l'origine de l'artère carotide interne et celles de sa terminaison étendue au premier segment de l'artère cérébrale moyenne et de l'artère cérébrale antérieure se reperméabilisent moins facilement que les occlusions d'une branche de l'artère cérébrale moyenne [21, 22]. II peut également survenir, dans un tiers des cas, une ré-occlusion artérielle après recanalisation initiale: ce risque est plus élevé en cas de recanalisa- tion partielle que complète, et est de mauvais pronostic puisqu'elle réduit de moitié les chances de guérison à trois mois [23]. Une thrombolyse trop tardive s'accompagne également d'un risque plus élevé d'échec: en effet, le bénéfice potentiel de la thrombolyse diminue rapidement au cours des premières heures, tandis qu'augmente le risque de transformation hémorragique grave. D'autres facteurs ont une action délétère sur la zone de pénombre: la pression artérielle et la baisse du débit sanguin cérébral dans la zone, ainsi que l'hyperglycémie et l'hyperthermie qui influencent défavorablement la tolérance à l'ischémie de cette zone. Par ailleurs, sont aussi causes d'échec une transformation hémorragique, qui survient chez $6 \%$ des patients [2], et l'absence de lyse du caillot ou une lyse seulement partielle, en raison de sa constitution biochimique. Enfin, d'autres facteurs incriminés dans l'échec de la thrombolyse, encore méconnus, font l'objet de différentes études [14].

\section{Thrombolyse intra-artérielle}

La fenêtre des trois heures limite le nombre des patients pouvant bénéficier du traitement par rt-PA en intraveineux. Des études antérieures ont montré que, avant la sixième heure, $80 \%$ des malades ont une occlusion artérielle cérébrale dans le territoire symptomatique [2]. D'où le handicap thérapeutique dans ce groupe de

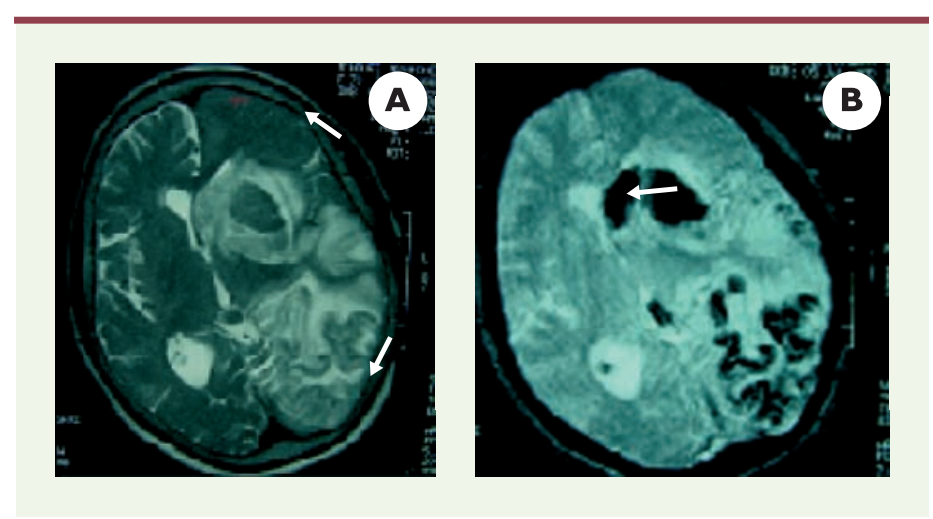

Figure 1. L'hémorragie intracrânienne est une complication possible de la thrombolyse. Patiente de 70 ans ayant présenté une hémiplégie droite massive avec une aphasie globale sur une occlusion de l'artère sylvienne gauche. Le scanner cérébral était normal. Un traitement par rt-PA $(0,9 \mathrm{mg} / \mathrm{kg})$ a été administré 2 h 50 après le début des symptômes. L'IRM de contrôle à 24 heures de la thrombolyse montrait un infarctus hémorragique diffus. Son score de Rankin à 3 mois était de 2 (aphasie résiduelle modérée). A. IRM coupe axiale séquence pondérée en T2: volumineux œdème hémisphérique (flèche) avec un effet de masse sur le ventricule latéral (flèche). B. IRM coupe axiale séquence pondérée en T2*: hyposignal serpigineux en frontopariétal gauche avec un hématome (flèche) au niveau de la tête du noyau caudé. 
patients arrivant au-delà de la fenêtre des trois heures. La thrombolyse intra-artérielle pourrait être bénéfique chez ces patients [24, 25]. Plusieurs séries de thrombolyse intra-artérielle indiquent qu'une reperméabilisation précoce du tronc basilaire, des artères vertébrales et de l'artère cérébrale moyenne peut conduire à une amélioration de la survie. Une bonne reperméabilisation était observée après cathétérisme hypersélectif et injection directe d'urokinase ou de rt-PA dans les 10 premières heures suivant la survenue des symptômes (Figure 2).

\section{Place de l'IRM cérébrale}

L'IRM multimodale permet une identification rapide des caractéristiques de l'infarctus cérébral (localisation, taille et nature de l'infarctus, présence d'une occlusion artérielle et étendue de la zone de pénombre) [25]. À terme, I'IRM permettrait de définir des seuils (hémodynamiques et parenchymateux) de moindre réponse à un traitement thrombolytique par voie intraveineuse. Elle permettra dans l'avenir de mieux sélectionner les candidats à la fibrinolyse, la détection rapide des candidats à une approche intra-artérielle et l'identification des patients susceptibles de bénéficier d'un traitement au-delà de la fenêtre thérapeutique des 3 heures [26, 27].

\section{Conclusions}

Il est clair que la thrombolyse ne peut être considérée comme un traitement universel de l'infarctus cérébral. Elle a eu pour intérêt d'entrầner des équipes d'urgences neurologiques à être plus entreprenantes en présence d'un accident vasculaire cérébral, compte tenu de la fenêtre «de trois heures».

La création de stroke-units devrait aboutir à un raccourcissement drastique des délais de prise en charge des malades, avec en corollaire une accélération de la mise en œuvre des nouvelles thérapeutiques et une meilleure sélection des groupes de patients susceptibles de bénéficier de ces médicaments. Un autre antithrombotique, le Réopro (inhibiteur de la GPIIb/IIla, le récepteur plaquettaire du fibrinogène) par voie intraveineuse, semble très intéressant dans les études préli-
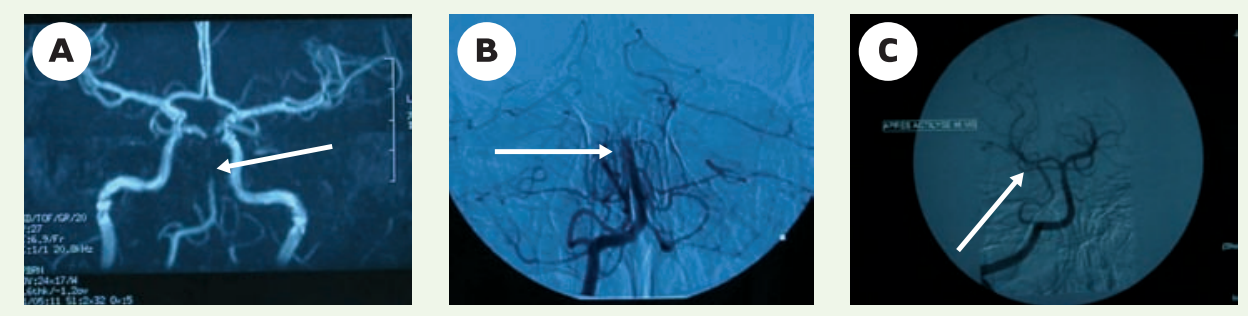

Figure 2. Reperméabilisation du tronc basilaire par thrombolyse intra-artérielle. Patiente de 24 ans ayant présenté une hémiparésie droite rapidement compliquée d'une ophtalmoplégie complexe et d'un trouble de la vigilance. Le bilan vasculaire avait montré une occlusion du tronc basilaire (TB). La patiente a subi une thrombolyse par voie intra-artérielle. Son score de Rankin à 3 mois était à 0 . A. Angiographie par résonance magnétique montrant une occlusion du TB (flèche) . B, C. Artériographie cérébrale confirmant l'occlusion du TB (flèche) (B) et la reperméabilisation du TB après thrombolyse (flèche) par voie intra-artérielle (C). minaires, et un grand essai est actuellement en cours pour l'évaluer en phase III. Quant à l'avenir de la prise en charge du patient frappé d'une ischémie cérébrale, il est comparable à celui de l'ischémie coronaire. Les patients localisés à proximité d'une unité neurovasculaire équipée d'une salle d'angiographie pourraient bénéficier d'une revascularisation en urgence par moyens mécaniques (capture du caillot, laser, stenting, etc.) ou par thrombolyse artérielle, ou d'une combinaison de ces deux traitements, suivie d'une perfusion veineuse d'inhibiteur de la GPIIb/IIla. Pour les autres centres, trop éloignés ou dépourvus d'hélicoptère, l'optimisation du système du soin et l'introduction de la télémédecine devraient permettre l'utilisation plus fréquente de la thrombolyse par rt-PA, suivie ou non d'une perfusion veineuse d'inhibiteur GPIIb/IIla, I'ensemble de ces stratégies thérapeutiques devant être convenablement évaluées.

Nous sommes au tout début du traitement de l'infarctus cérébral. Mais il est certain que la complexité et la diversité de ses causes et mécanismes exigent l'intervention de neurologues entraînés, équipés d'IRM disponibles « 24 h sur 24 h », de matériel d'examen par ultrasons et d'une table d'angiographie. $\diamond$

\section{SUMMARY}

\section{Thrombolysis and acute stroke}

Thrombolytic therapy are the most important advance in the management of acute ischemic stroke and has been evaluated in several randomised trials. Thrombolysis with recombinant tissue plasminogen activator ( $r t-P a)$ is effective within $3 \mathrm{~h}$ of onset of ischemic stroke and this efficacy is similar between different stroke subtypes. New trials will determine if extension of this timewindow can be substantiated. Therapy beyond the 3-hour window, with intra-arterial thrombolysis, appears to improve outcome but are applicable to selected group of patients. Thrombolytic drugs can also carry an important risk ( $5 \%$ to $10 \%$ ) of brain hemorrhage and edema that can prove fatal. The risk of symptomatic intracranial hemorrhage is directly proportional to stroke severity 
and inversely proportional to time to treatment. There is a growing interest in the use of MRI in acute ischemic stroke. It helps identify location of early cerebral ischemia and provides valuable information not only of the penumbra but also of vessel occlusion. Its use might help in selecting patients who will benefit most from treatment such as thrombolysis. In spite of these results, community use of thrombolytic therapy remains dismally low. Many physicians and medical centers are not presently equipped or willing to give thrombolytic drugs for stroke treatment. Increasing stroke awareness in the community, creating stroke unit and physicians education are necessary to extend the effective use of acute treatment in cerebral infarct to a larger group of patients. $\Delta$

\section{RÉFÉRENCES}

1. Larrue V, Amarenco P, Caussanel JP, et al. Recommendations for the use of intravenous thrombolytic therapy in cerebrovascular ischemic accident. French society of neurovascular disorders. Rev Neurol (Paris) 2000; 156: 1178-85.

2. The National institute of neurological disorders and stroke rt-PA stroke study group. Tissue plasminogen activator for acute ischemic stroke. $N$ Engl J Med 1995; 333: 1581-7.

3. Marler JR, Tilley BC, Lu M, et al. Early stroke treatment associated with better outcome: the NINDS rt-PA stroke study. Neurology 2000; 55: 1649-55.

4. Hacke W, Kaste M, Fieschi C, et al. Intravenous thrombolysis with recombinant tissue plasminogen activator for acute hemispheric stroke. The European cooperative acute stroke study (ECASS). JAMA 1995; 274: 1017-25.

5. Hacke W, Kaste M, Fieschi C, et al. Randomised double-blind placebo-controlled trial of thrombolytic therapy with intravenous alteplase in acute ischaemic stroke (ECASS II). Second european-australasian acute stroke study investigators. Lancet $1998 ; 352$ : 1245-51.

6. Clark WM, Wissman S, Albers GW, et al. Recombinant tissue-type plasminogen activator (Alteplase) for ischemic stroke 3 to 5 hours after symptom onset. The ATLANTIS study: a randomized controlled trial. Alteplase thrombolysis for acute noninterventional therapy in ischemic stroke. JAMA 1999; 282: 2019-26.

7. Randomised controlled trial of streptokinase, aspirin, and combination of both in treatment of acute ischaemic stroke. Multicentre acute stroke trial-Italy (MAST-I) group. Lancet 1995; 346: 1509-14.

8. Thrombolytic therapy with streptokinase in acute ischemic stroke. The multicenter acute stroke trial-Europe study group. N Engl I Med 1996; 335: 145-50.

9. Donnan GA, Davis SM, Chambers BR, et al. Trials of streptokinase in severe acute ischaemic stroke. Lancet 1995; 345: 578-9.

10. Hacke W, Donnan G, Fieschi C, et al. Association of outcome with early stroke treatment: pooled analysis of ATLANTIS, ECASS, and NINDS rt-PA stroke trials. Lancet $2004 ; 363: 768-74$.
11. Wardlaw JM, Warlow CP, Counsell C. Systematic review of evidence on thrombolytic therapy for acute ischaemic stroke. Lancet 1997; 350: 607-14.

12. Molina CA, Montaner J, Abilleira S, et al. Timing of spontaneous recanalization and risk of hemorrhagic transformation in acute cardioembolic stroke. Stroke 2001; 32 : 1079-84.

13. Larrue V, von Kummer RR, Muller A, Bluhmki $\varepsilon$. Risk factors for severe hemorrhagic transformation in ischemic stroke patients treated with recombinant tissue plasminogen activator: a secondary analysis of the European-Australasian acute stroke study (ECASS II). Stroke 2001; 32: 438-41.

14. Selim M, Fink JN, Kumar $S$, et al. Predictors of hemorrhagic transformation after intravenous recombinant tissue plasminogen activator: prognostic value of the initial apparent diffusion coefficient and diffusion-weighted lesion volume. Stroke 2002; 33: 2047-52.

15. Knight RA, Dereski MO, Helpern JA, et al. Magnetic resonance imaging assessment of evolving focal cerebral ischemia. Comparison with histopathology in rats. Stroke $1994 ; 25: 1252-62$

16. Miyasaka N, Nagaoka T, Kuroiwa T, et al. Histopathologic correlates of tempora diffusion changes in a rat model of cerebral hypoxia/ischemia. Am J Neuroradiol $2000 ; 21: 60-6$.

17. Del Zoppo GJ, von Kummer R, Hamann GF. Ischaemic damage of brain microvessels: inherent risks for thrombolytic treatment in stroke.J Neurol Neurosurg Psychiatry $1998 ; 65: 1-9$.

18. Dijkhuizen RM, Asahi M, Wu 0, et al. Delayed rt-PA treatment in a rat embolic stroke model: diagnosis and prognosis of ischemic injury and hemorrhagic transformation with magnetic resonance imaging. J Cereb Blood Flow Metab 2001; 21: 964-71.

19. Ueda T, Hatakeyama T, Kumon Y, et al. Evaluation of risk of hemorrhagic transformation in local intra-arterial thrombolysis in acute ischemic stroke by initial SPECT. Stroke 1994; 25 : 298-303.

20. Shimosegawa $\varepsilon$, Hatazawa J, Inugami A, et al. Cerebral infarction within six hours of onset: prediction of completed infarction with technetium-99m-HMPAO SPECT. J Nucl Med 1994; 35 : 1097-103

21. Del Zoppo GJ, Poeck K, Pessin MS, et al. Recombinant tissue plasminogen activator in acute thrombotic and embolic stroke. Ann Neurol 1992; 32: 78-86.

22. Mori $\varepsilon$, Yoneda $Y$, Tabuchi $M$, et al. Intravenous recombinant tissue plasminogen activator in acute carotid artery territory stroke. Neurology 1992; 42: 976-82.

23. Alexandrov AV, Grotta JC. Arterial reocclusion in stroke patients treated with intravenous tissue plasminogen activator. Neurology 2002; 59: 862-7.

24. Furlan A, Higashida R, Wechsler L, et al. Intra-arterial prourokinase for acute ischemic stroke. The PROACT II study: a randomized controlled trial. Prolyse in acute cerebral thromboembolism. JAMA 1999; 282: 2003-11.

25. Hacke W, Warach S. Diffusion-weighted MRI as an evolving standard of care in acute stroke. Neurology 2000; 54 : 1548-9.

26. Parsons MW, Barber PA, Chalk J, et al. Diffusion and perfusion-weighted MRI response to thrombolysis in stroke. Ann Neurol 2002; 51: 28-37.

27. Kidwell CS, Saver JL, Mattiello J, et al. Thrombolytic reversal of acute human cerebral ischemic injury shown by diffusion/perfusion magnetic resonance imaging. Ann Neurol $2000 ; 47: 462-9$.

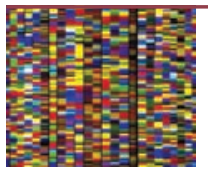

Retrouvez chaque mois médecine/sciences

sur France-Info dans la chronique « Info-Sciences 》

de Marie-Odile Monchicourt, du lundi au mercredi.

france-info.com 